\title{
Algebraic Type Approximation to the Blasius Velocity Profile
}

\author{
Beong In Yun \\ Department of Statistics and Computer Science, Kunsan National University, Gunsan 573-701, Republic of Korea
}

Correspondence should be addressed to Beong In Yun; paulllyun@gmail.com

Received 17 December 2013; Revised 18 February 2014; Accepted 25 February 2014; Published 24 March 2014

Academic Editor: Mohamad Alwash

Copyright (C) 2014 Beong In Yun. This is an open access article distributed under the Creative Commons Attribution License, which permits unrestricted use, distribution, and reproduction in any medium, provided the original work is properly cited.

For the Blasius velocity profile we propose a simple algebraic type approximate function which is uniformly accurate over the whole region. Moreover, for further improvement a correction method based on a weight function is introduced. The availability of the proposed method is shown by the result of numerical experiments.

\section{Introduction}

We consider the well-known Blasius problem

$$
N f(x):=2 f^{\prime \prime \prime}(x)+f(x) f^{\prime \prime}(x)=0, \quad 0 \leq x<\infty,
$$

subject to the boundary conditions

$$
f(0)=f^{\prime}(0)=0, \quad f^{\prime}(\infty)=1 .
$$

The so-called Blasius function $f(x)$ describes the stream on the boundary layer over a flat plate. There are lots of analytical approximation methods to the Blasius function $f(x)$ such as the variational iteration method [1-6], the Adomian decomposition method [7-9], and the homotopy analysis method [10-12]. Recently, spectral methods based on orthogonal functions have been applied in approximation of solutions for the nonlinear boundary value problems like the Blasius problem [13-17]. In addition, numerical solutions of the nonlinear differential equations for the boundary layer problems such as Falkner-Skan equations, including the Blasius equation as a special case, have been studied by many researchers [18-25].

Concerning the streamwise velocity profile $f^{\prime}(x)$, we note an approximate analytical solution proposed in the literature [26] of the form

$$
\begin{aligned}
f_{Y}^{\prime}(x)= & \tanh (b x) \\
& +2 c r \operatorname{sech}^{2}(r x)\left\{\tanh ^{3}(r x)+2 \tanh ^{7}(r x)\right\},
\end{aligned}
$$

where $b$ and $c$ are determined by the known properties of the Blasius function $f(x)$ at the wall $x=0$ and far from the wall, respectively. The parameter $r$ is chosen by minimizing the residual function

$$
N f_{Y}(x)=2 f_{Y}^{\prime \prime \prime}(x)+f_{Y}(x) f_{Y}^{\prime \prime}(x) .
$$

Recently, Savaş [27] introduced another approximate analytical solution for the streamwise velocity profile as

$$
f_{S, n}^{\prime}(x)=\left(\tanh \left[(\alpha x)^{n}\right]\right)^{1 / n}
$$

for the constants $(\alpha, n)=(0.33206,3 / 2)$ or $(0.33245,5 / 3)$.

In the next section, motivated by the analytical solutions (3) and (5), we propose another algebraic type approximate analytical solution for the velocity profile as given by (6) and explore its properties with a method to determine the parameters therein. In Section 3, by using an appropriate weight function, we introduce a correction method to improve the accuracy of the presented approximation. Moreover, for further improvement we employ an auxiliary term which appropriately reflects the error of the presented approximation. Some numerical experiments are performed to demonstrate the efficiency of the presented method.

\section{Approximation to the Velocity Profile}

To approximate the velocity profile $f^{\prime}(x)$ directly we suggest an algebraic type analytical function as

$$
f_{A, m}^{\prime}(x)=\left[\frac{(a x)^{m}}{(a x)^{m}+1}\right]^{1 / m}
$$


for a constant $a>0$ and an exponent $m>1$. We note that $f_{A, m}^{\prime}$ satisfies the boundary conditions $f_{A, m}^{\prime}(0)=0$ and $f_{A, m}^{\prime}(\infty)=$ 1 given in (2), and its derivative is

$$
f_{A, m}^{\prime \prime}(x)=\frac{f_{A, m}^{\prime}(x)}{x\left\{(a x)^{m}+1\right\}}=\frac{a}{\left\{(a x)^{m}+1\right\}^{1+(1 / m)}} .
$$

Since $f_{A, m}^{\prime \prime}(0)=a$, we may set

$$
a=f^{\prime \prime}(0)=0.332057 \ldots
$$

which is a well-known Blasius constant [28]. In addition, the velocity profile $y=f_{A, m}^{\prime}(x)$ has an inversion of a simple form as

$$
x=\frac{1}{a}\left[\frac{y^{m}}{1-y^{m}}\right]^{1 / m}, \quad 0 \leq y<1 .
$$

The related approximation $f_{A, m}$ to the Blasius stream function $f(x)$ can be obtained by the formula

$$
f_{A, m}(x)=\int_{0}^{x} f_{A, m}^{\prime}(t) d t=\int_{0}^{x}\left[\frac{(a t)^{m}}{(a t)^{m}+1}\right]^{1 / m} d t .
$$

In fact, using the symbolic computational software Mathematica (version 9), one can find the analytical form of $f_{A, m}$ as

$$
f_{A, m}(x)=\frac{a}{2} x^{2} F\left(\frac{1}{m}, \frac{2}{m}, \frac{m+2}{m} ;-(a x)^{m}\right),
$$

where $F(p, q, r ; z)$ is the hypergeometric function [29] whose series expansion is

$$
F(p, q, r ; z)=\sum_{k=0}^{\infty} \frac{(p)_{k}(q)_{k}}{(r)_{k} k !} z^{k}
$$

and $(s)_{k}$ is the shifted factorial defined by

$$
(s)_{k}=s(s+1)(s+2) \cdots(s+k-1), \quad k \geq 1
$$

with $(s)_{0}=1$.

For an appropriate parameter $m$ in $f_{A, m}^{\prime}(x)$ we may choose a value $m=m^{*}$ at which the $L_{2}$-norm of the residual function $N f_{A, m}$,

$$
\left\|N f_{A, m}\right\|_{2}^{2}=\int_{0}^{\infty}\left\{N f_{A, m}(x)\right\}^{2} d x
$$

is minimized. To find $m^{*}$ one can use a package, Mathematica, for example, and we will obtain the local minimum in $\left\|N f_{A, m}\right\|_{2}$ at the value $m^{*} \approx 4.216$.

Figure 1 shows the errors of the presented approximate velocity profile, $f_{A, m}^{\prime}(x)$, with integers $m=4$ and $m=5$ near the value $m^{*} \approx 4.216$. The error means difference between $f_{A, m}^{\prime}(x)$ and the numerical solution for the velocity profile $f^{\prime}(x)$ which is regarded as an exact solution. By numerical experiments for various values of $m$, we can see that the accuracy of $f_{A, m}^{\prime}(x)$ becomes better far from the wall $x=0$ as $m$ goes large while it becomes better near $x=0$ as $m$ goes small.

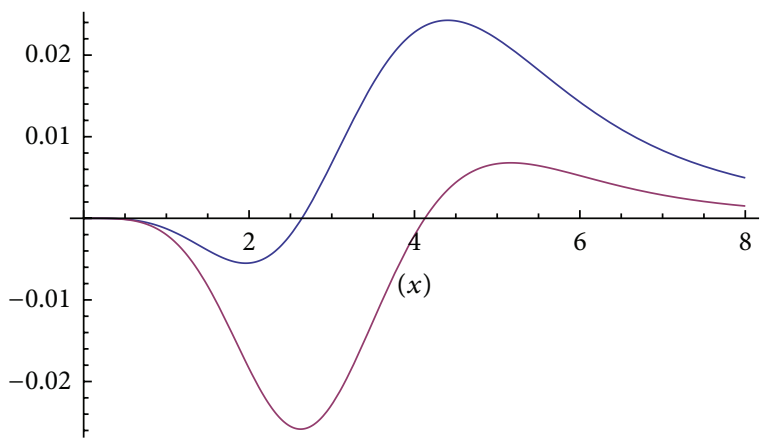

FIGURE 1: Errors of the presented approximate velocity profiles, $f_{A, 4}^{\prime}(x)$ (upper line) and $f_{A, 5}^{\prime}(x)$ (lower line).

\section{Improvement by a Weighted Average}

In order to improve the accuracy of the proposed approximate velocity profile over the whole region, we introduce a weighted average

$$
\tilde{f}_{A, m, n}^{\prime}(x)=(1-\theta(x)) f_{A, m}^{\prime}(x)+\theta(x) f_{A, n}^{\prime}(x)
$$

for $1<m<n$, where $\theta(x)$ is a weight function defined as

$$
\theta(x)=\theta(b, k ; x)=\frac{(x / b)^{k}}{(x / b)^{k}+1}, \quad 0 \leq x<\infty
$$

for $b>0$ and $k>0$. It follows that $0 \leq \theta(x)<1$ for $0 \leq x<\infty$ with $\theta(b)=1 / 2$. Moreover, it should be noticed that for a sufficiently large $k$

$$
\theta(x) \sim \begin{cases}0, & \text { for } x<b \\ 1, & \text { for } x>b\end{cases}
$$

and thus

$$
\tilde{f}_{A, m, n}^{\prime}(x) \sim \begin{cases}f_{A, m}^{\prime}(x), & \text { for } x<b, \\ f_{A, n}^{\prime}(x), & \text { for } x>b\end{cases}
$$

This implies that the point $x=b$ plays the role of $a$ threshold between two approximate velocity profiles $f_{A, m}^{\prime}$ and $f_{A, n}^{\prime}$. On the other hand, the related approximate stream function $\tilde{f}_{A, m, n}$ can be obtained by numerical integration in the equation

$$
\tilde{f}_{A, m, n}(x)=\int_{0}^{x} \tilde{f}_{A, m, n}^{\prime}(t) d t .
$$

Referring to Figure 1 for the cases of $m=4$ and $n=5$, we may take $b=3.5134$ in (16) which is a center of the points $x=$ 4.4033 and 2.6234 at which $f_{A, 4}(x)$ and $f_{A, 5}(x)$, respectively, have the maximum absolute errors. Thick lines in Figure 2 indicate errors (i.e., differences from the numerical solution) of the corrected approximate stream function $\widetilde{f}_{A, 4,5}(x)$ and the velocity profile $\tilde{f}_{A, 4,5}^{\prime}(x)$ with $b=3.5134$ and $k=6$ in the weight function $\theta(x)=\theta(b, k ; x)$. We can see that the maximum error is about 0.01 in the velocity profile and 


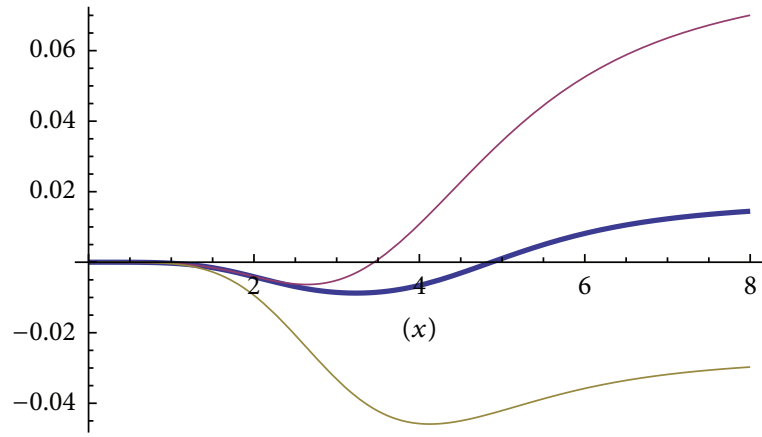

(a)

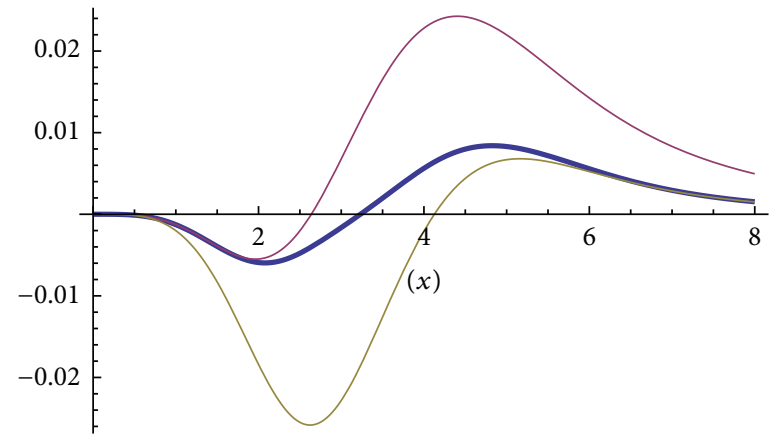

(b)

FIGURE 2: Errors of the corrected approximate stream function $\widetilde{f}_{A, 4,5}(x)$ (in (a)) and the velocity profile $\widetilde{f}_{A, 4,5}^{\prime}(x)$ (in (b)), indicated by thick lines. In addition, errors of $f_{A, 4}(x)$ and $f_{A, 5}(x)$ and those of $f_{A, 4}^{\prime}(x)$ and $f_{A, 5}^{\prime}(x)$ are, respectively, included in (a) and (b), indicated by thin lines.
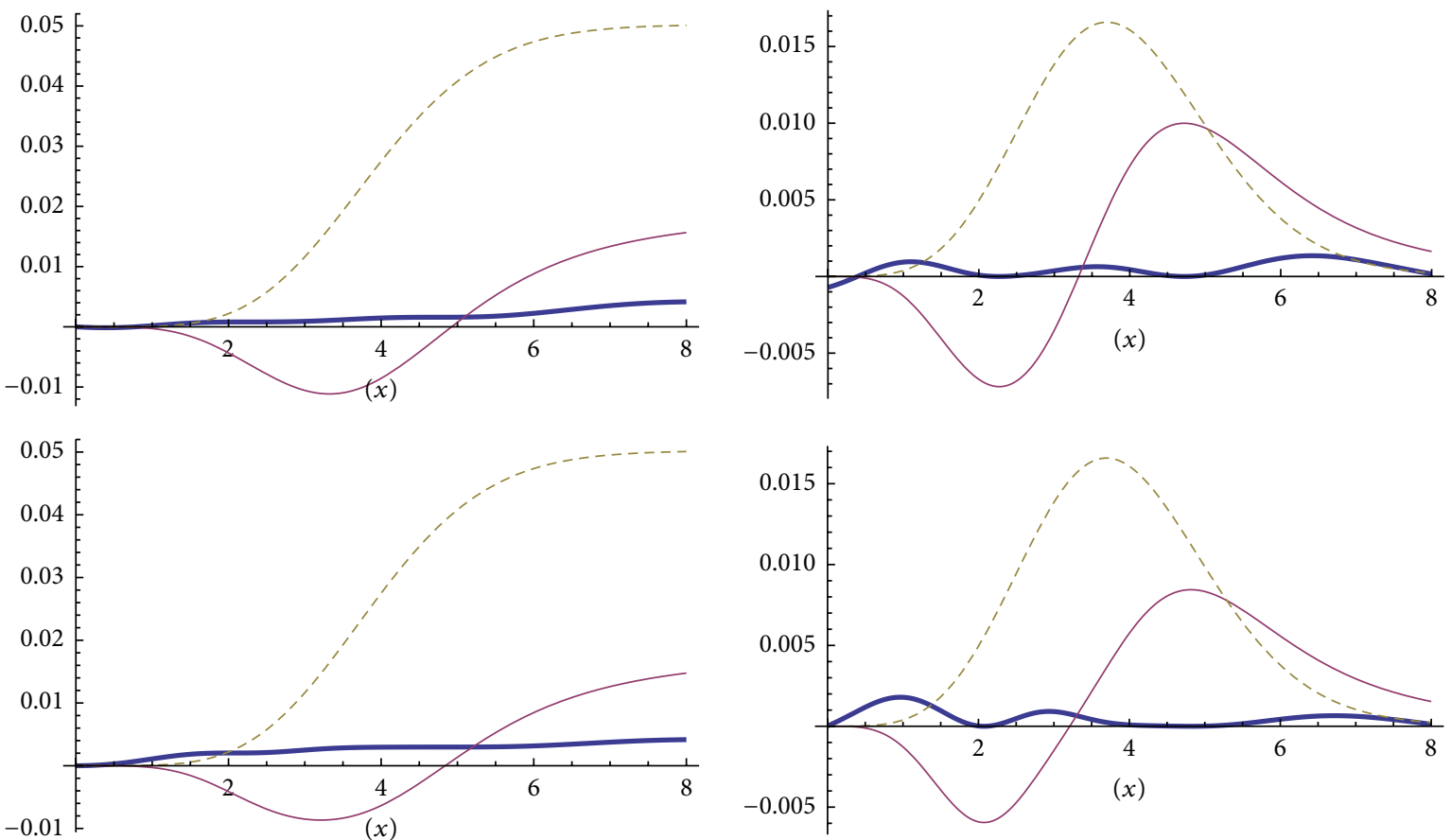

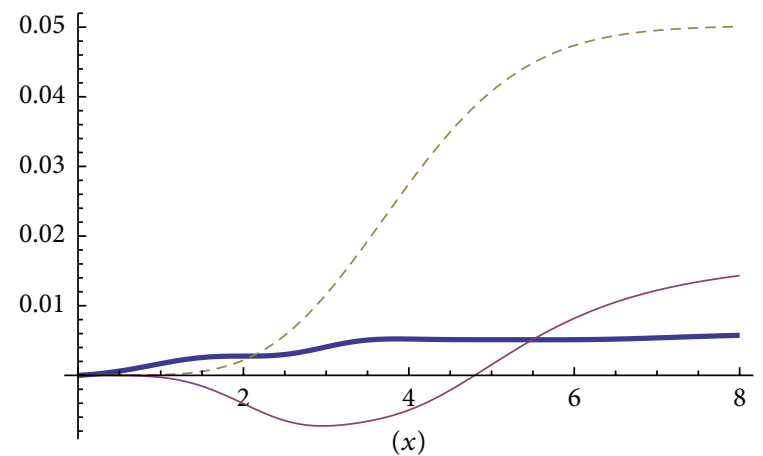

(a)

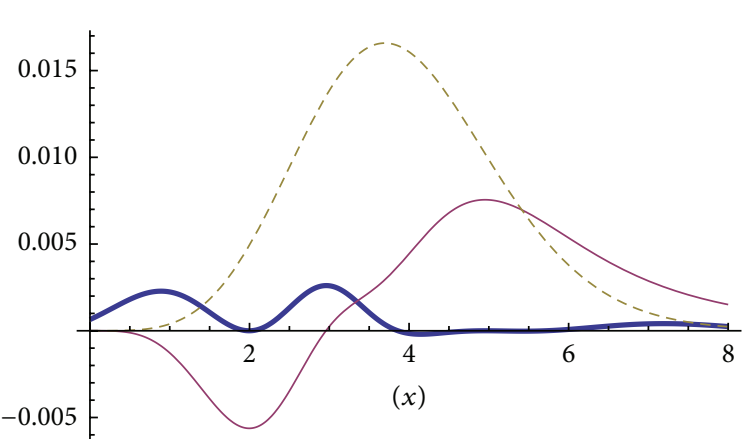

(b)

FIGURE 3: Errors of the corrected approximate stream function $\widehat{f}_{A, 4,5}(x)$ (in (a)) and the velocity profile $\widehat{f}_{A, 4,5}^{\prime}(x)$ (in (b)), indicated by thick lines, for the parameters $k=4,6,8$ from the top row. Thin lines indicate errors of $\widetilde{f}_{A, 4,5}(x)$ and $\widetilde{f}_{A, 4,5}^{\prime}(x)$ and dashed lines indicate Savas's approximations $f_{S, 1.5}(x)$ and $f_{S, 1.5}^{\prime}(x)$. 
TABLE 1: Numerical values of the constants $c_{1}, c_{2}, M_{1}$, and $M_{2}$ in (20) for the parameters $3 \leq k \leq 8$.

\begin{tabular}{ccccc}
\hline$k$ & $c_{1}$ & $c_{2}$ & $M_{1}$ & $M_{2}$ \\
\hline 3 & 2.3510 & 4.7054 & -0.008444 & 0.011006 \\
4 & 2.2706 & 4.7209 & -0.007185 & 0.009994 \\
5 & 2.1595 & 4.7584 & -0.006378 & 0.009134 \\
6 & 2.0739 & 4.8167 & -0.005948 & 0.008443 \\
7 & 2.0241 & 4.8859 & -0.005734 & 0.007923 \\
8 & 1.9964 & 4.9526 & -0.005624 & 0.007553 \\
\hline
\end{tabular}

about 0.02 in the stream function. Comparing these with the errors of the approximate stream functions $f_{A, 4}(x)$ and $f_{A, 5}(x)$ in Figure 2(a) and the velocity profiles $f_{A, 4}^{\prime \prime}(x)$ and $f_{A, 5}^{\prime \prime}(x)$ in Figure 2(b), one can find distinct improvement of the corrected velocity profile $\widetilde{f}_{A, 4,5}^{\prime}(x)$ defined in (15).

In practice, by numerical experiments, we can find better case of parameters like $(m, n)=(3.8,5.8)$, for example, which results in more accurate approximation with the maximum errors about 0.003 and 0.005 in the velocity profile and the stream function, respectively. However, this choice of the parameters looks rather ambiguous. Thus, for development of plausible further improvement, we refer to the correction method proposed in the literature [30] which uses an auxiliary term reflecting the error of the presented approximation. First, observing the behavior of the error $E(x)=f^{\prime}(x)-$ $\tilde{f}_{A, m, n}^{\prime}(x)$ given in Figure 2, for example, we can have the numerical values of the critical points $\left(c_{1}, M_{1}\right)=\left(c_{1}, E\left(c_{1}\right)\right)$ and $\left(c_{2}, M_{2}\right)=\left(c_{2}, E\left(c_{2}\right)\right)$ of $E(x)$. Then, to approximate $E(x)$ appropriately, we suggest a function $g_{m, n}(x) \approx E(x)$ of the form

$$
g_{m, n}(x)=M(x-c) e^{-r(x-c)^{2}}+\bar{M},
$$

where

$$
\begin{gathered}
M=\frac{M_{2}-M_{1}}{2}, \quad \bar{M}=\frac{M_{1}+M_{2}}{2}, \\
c=\frac{c_{1}+c_{2}}{2} .
\end{gathered}
$$

The value of $r$ in (20) can be determined by the condition $g_{m, n}^{\prime}\left(c_{1}\right)=g_{m, n}^{\prime}\left(c_{2}\right)=0$ which implies that

$$
r=\frac{2}{\left(c_{2}-c_{1}\right)^{2}} \text {. }
$$

We consider a corrected approximation

$$
\hat{f}_{A, m, n}^{\prime}(x)=\tilde{f}_{A, m, n}^{\prime}(x)+g_{m, n}(x) .
$$

One may expect that the accuracy of $\widehat{f}_{A, m, n}^{\prime}(x)$ goes higher as $g_{m, n}(x)$ becomes closer to the error $E(x)$.

For example, for the case of $(m, n, k)=(4,5, k), 3 \leq k \leq 8$, we can evaluate numerical values of the constants $c_{1}, c_{2}, M_{1}$, and $M_{2}$ as given in Table 1. Figure 3 shows errors of $\widehat{f}_{A, 4,5}(x)$ and the velocity profile $\widehat{f}_{A, 4,5}^{\prime}(x)$ indicated by thick lines, compared with those of $\tilde{f}_{A, 4,5}(x)$ and $\tilde{f}_{A, 4,5}^{\prime}(x)$ indicated by thin lines, for the parameters $k=4,6,8$. Additionally, dashed lines indicate Savas's approximations $f_{S, 1.5}(x)$ and $f_{S, 1.5}^{\prime}(x)$. We can find that $\widehat{f}_{A, 4,5}^{\prime}(x)$ and $\widehat{f}_{A, 4,5}(x)$ with $k=4,6$ have the maximum errors about 0.002 and 0.005 , respectively. This implies that the correction method (23) can highly improve the proposed method (15) as a result.

\section{Conflict of Interests}

The author declares that there is no conflict of interests regarding the publication of this paper.

\section{Acknowledgment}

This research was supported by the Basic Science Research Program through the National Research Foundation of Korea (NRF-2013R1A1A4A03005079).

\section{References}

[1] J. H. He, "Approximate analytical solution of Blasius' equation," Communications in Nonlinear Science and Numerical Simulation, vol. 3, no. 4, pp. 260-263, 1998.

[2] J. H. He, "A review on some new recently developed nonlinear analytical techniques," International Journal of Nonlinear Sciences and Numerical Simulation, vol. 1, no. 1, pp. 51-70, 2000.

[3] J. H. He, "A simple perturbation approach to Blasius equation," Applied Mathematics and Computation, vol. 140, no. 2-3, pp. 217-222, 2003.

[4] J. Lin, "A new approximate iteration solution of Blasius equation," Communications in Nonlinear Science \& Numerical Simulation, vol. 4, no. 2, pp. 91-99, 1999.

[5] J. Y. Parlange, R. D. Braddock, and G. Sander, "Analytical approximations to the solution of the Blasius equation," Acta Mechanica, vol. 38, no. 1-2, pp. 119-125, 1981.

[6] A.-M. Wazwaz, "The variational iteration method for solving two forms of Blasius equation on a half-infinite domain," Applied Mathematics and Computation, vol. 188, no. 1, pp. 485491, 2007.

[7] G. Adomian, "A review of the decomposition method in applied mathematics," Journal of Mathematical Analysis and Applications, vol. 135, no. 2, pp. 501-544, 1988.

[8] G. Adomian, "Solution of the Thomas-Fermi equation," Applied Mathematics Letters, vol. 11, no. 3, pp. 131-133, 1998.

[9] J. Biazar, M. Gholami Porshokuhi, and B. Ghanbari, "Extracting a general iterative method from an Adomian decomposition method and comparing it to the variational iteration method," Computers \& Mathematics with Applications, vol. 59, no. 2, pp. 622-628, 2010.

[10] F. M. Allan and M. I. Syam, "On the analytic solutions of the nonhomogeneous Blasius problem," Journal of Computational and Applied Mathematics, vol. 182, no. 2, pp. 362-371, 2005.

[11] S.-J. Liao, "A uniformly valid analytic solution of twodimensional viscous flow over a semi-infinite flat plate," Journal of Fluid Mechanics, vol. 385, pp. 101-128, 1999.

[12] S.-J. Liao, "An explicit, totally analytic approximate solution for Blasius' viscous flow problems," International Journal of NonLinear Mechanics, vol. 34, no. 4, pp. 759-778, 1999. 
[13] W. Bao and J. Shen, "A generalized-Laguerre-Hermite pseudospectral method for computing symmetric and central vortex states in Bose-Einstein condensates," Journal of Computational Physics, vol. 227, no. 23, pp. 9778-9793, 2008.

[14] K. Parand, M. Shahini, and M. Dehghan, "Rational Legendre pseudospectral approach for solving nonlinear differential equations of Lane-Emden type," Journal of Computational Physics, vol. 228, no. 23, pp. 8830-8840, 2009.

[15] K. Parand, M. Dehghan, A. R. Rezaei, and S. M. Ghaderi, "An approximation algorithm for the solution of the nonlinear LaneEmden type equations arising in astrophysics using Hermite functions collocation method," Computer Physics Communications, vol. 181, no. 6, pp. 1096-1108, 2010.

[16] K. Parand, M. Dehghan, and A. Taghavi, "Modified generalized Laguerre function tau method for solving laminar viscous flow: the Blasius equation," International Journal of Numerical Methods for Heat \& Fluid Flow, vol. 20, no. 6-7, pp. 728-743, 2010.

[17] J. Shen and L.-L. Wang, "Some recent advances on spectral methods for unbounded domains," Communications in Computational Physics, vol. 5, no. 2-4, pp. 195-241, 2009.

[18] S. Abbasbandy, "A numerical solution of Blasius equation by Adomian's decomposition method and comparison with homotopy perturbation method," Chaos, Solitons and Fractals, vol. 31, no. 1, pp. 257-260, 2007.

[19] A. Asaithambi, "Numerical solution of the Falkner-Skan equation using piecewise linear functions," Applied Mathematics and Computation, vol. 159, no. 1, pp. 267-273, 2004.

[20] I. Hashim, "Comments on a new algorithm for solving classical Blassius equation," Applied Mathematics and Computation, vol. 176, no. 2, pp. 700-703, 2006.

[21] L. Howarth, "On the solution of the laminar boundary layer equations," Proceedings of the Royal Society of London A, vol. 164, pp. 547-579, 1938.

[22] S. J. Liao, "A non-iterative numerical approach for the twodimensional viscous flow problems governed by the FalknerSkan equation," International Journal for Numerical Methods in Fluids, vol. 35, pp. 495-518, 2001.

[23] C.-S. Liu and J.-R. Chang, "The Lie-group shooting method for multiple-solutions of Falkner-Skan equation under suctioninjection conditions," International Journal of Non-Linear Mechanics, vol. 43, no. 9, pp. 844-851, 2008.

[24] L.-T. Yu and C.-K. Chen, "The solution of the Blasius equation by the differential transformation method," Mathematical and Computer Modelling, vol. 28, no. 1, pp. 101-111, 1998.

[25] L. Wang, "A new algorithm for solving classical Blasius equation," Applied Mathematics and Computation, vol. 157, no. 1, pp. $1-9,2004$.

[26] B. I. Yun, "Approximate analytical solutions using hyperbolic functions for the generalized Blasius problem," Abstract and Applied Analysis, vol. 2012, Article ID 581453, 10 pages, 2012.

[27] Ö. Savaş, "An approximate compact analytical expression for the Blasius velocity profile," Communications in Nonlinear Science and Numerical Simulation, vol. 17, no. 10, pp. 3772-3775, 2012.

[28] J. P. Boyd, "The Blasius function: computations before computers, the value of tricks, undergraduate projects, and open research problems," SIAM Review, vol. 50, no. 4, pp. 791-804, 2008.

[29] R. Beals and R. Wong, Special Functions, Cambridge University Press, New York, NY, USA, 1st edition, 2010.

[30] B. I. Yun, "Constructing uniform approximate analytical solutions for the Blasius problem," Abstract and Applied Analysis, vol. 2014, Article ID 495734, 6 pages, 2014. 


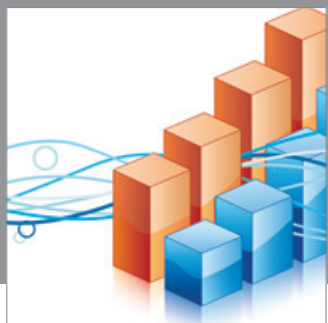

Advances in

Operations Research

mansans

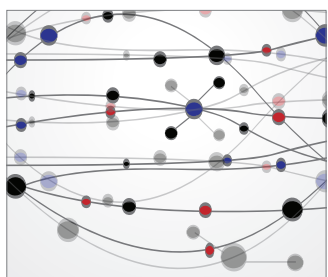

The Scientific World Journal
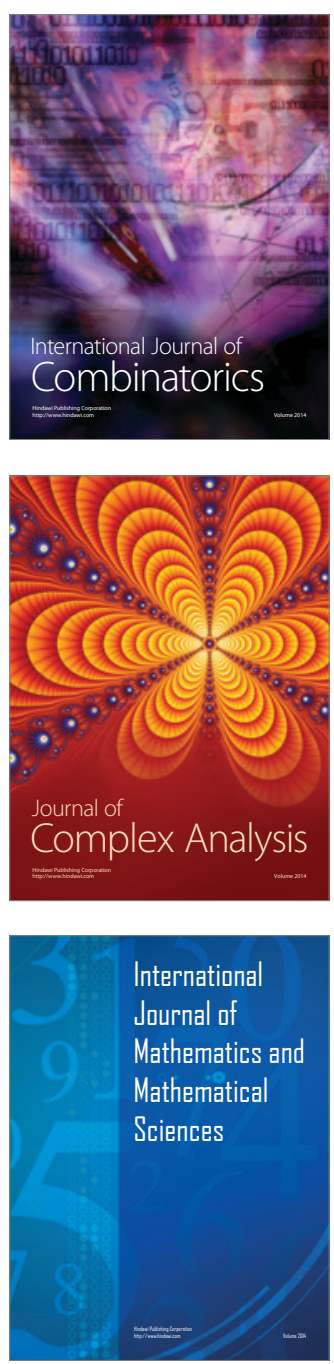
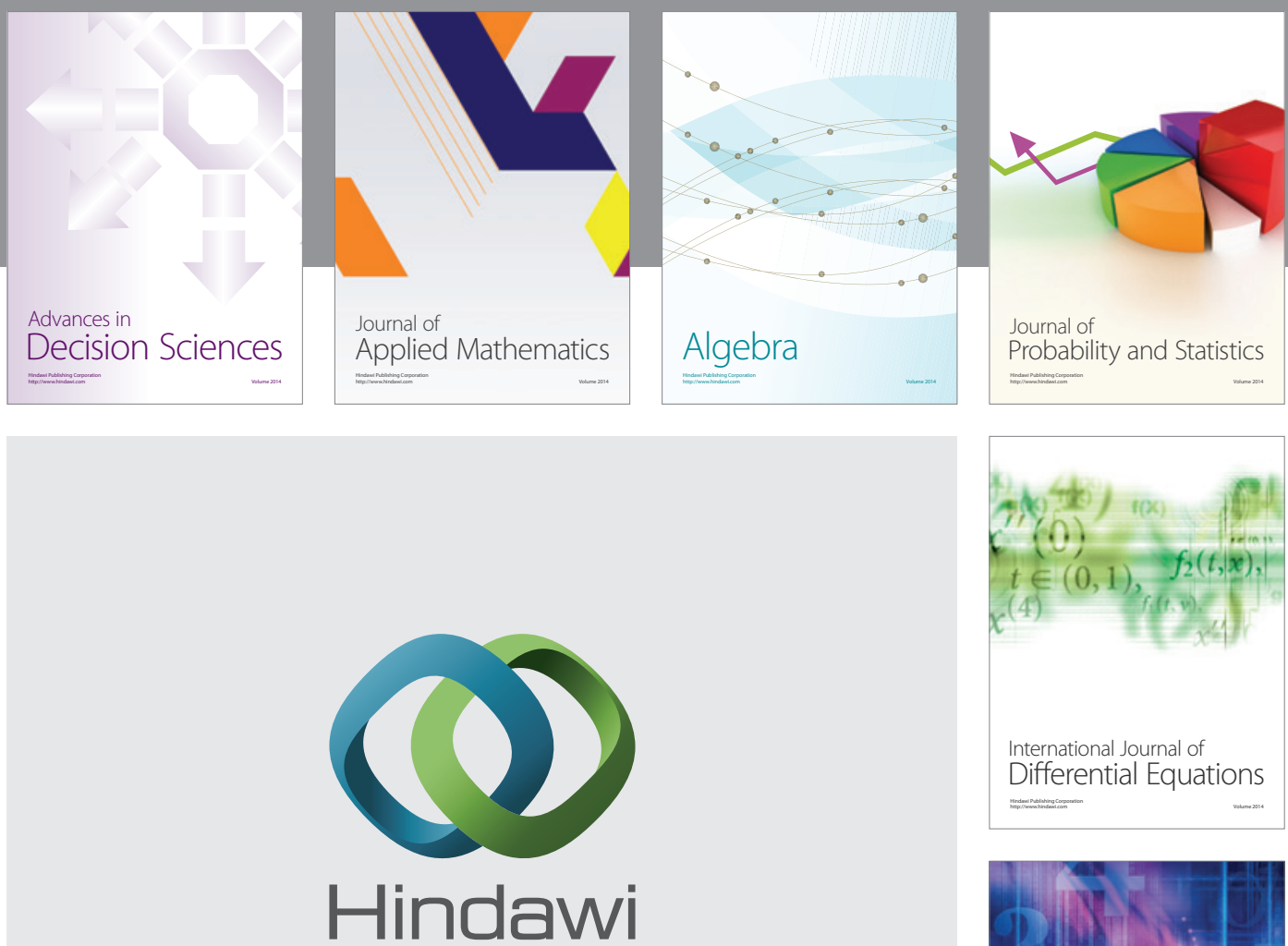

Submit your manuscripts at http://www.hindawi.com
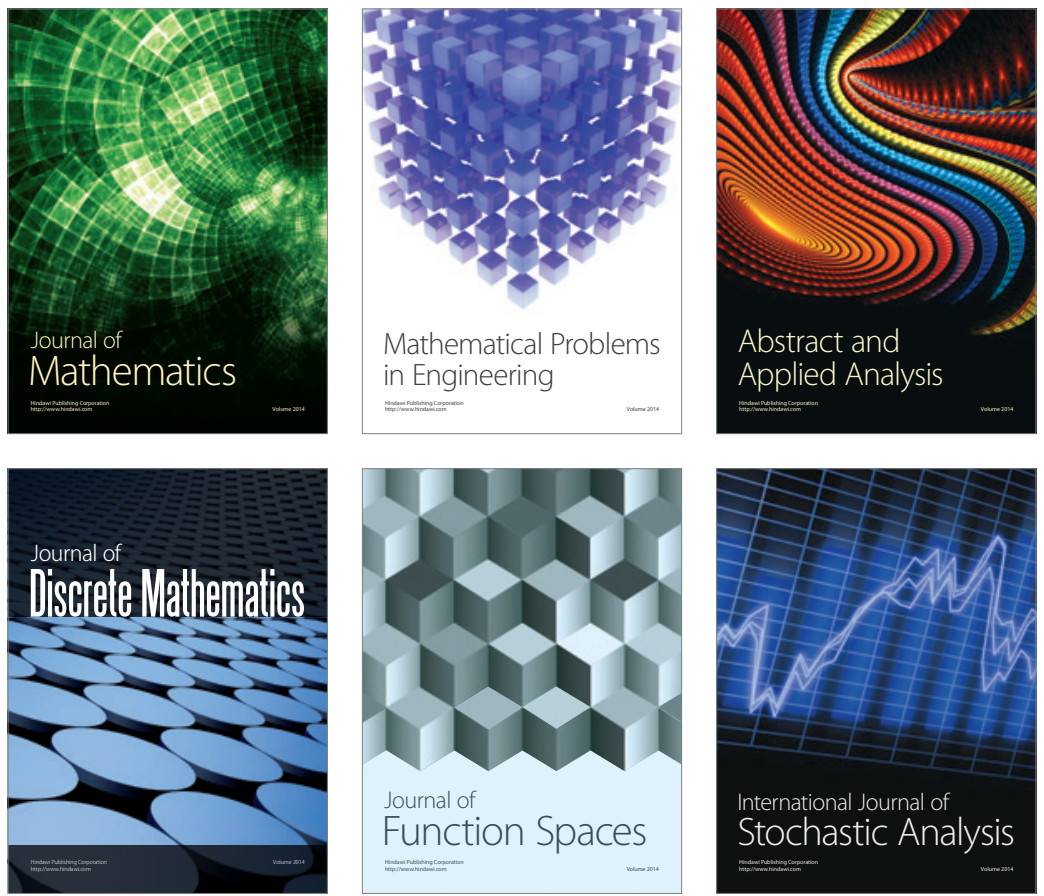

Journal of

Function Spaces

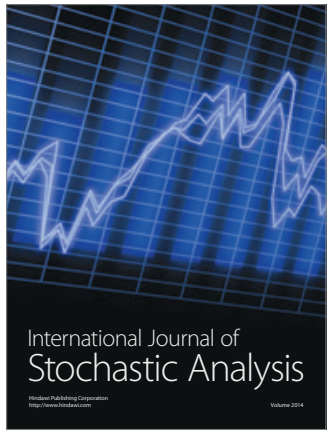

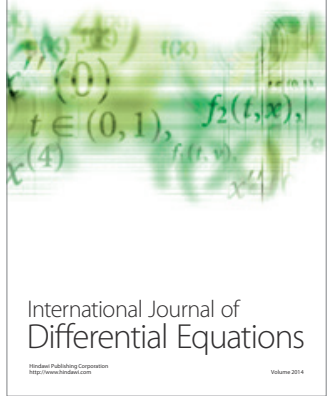
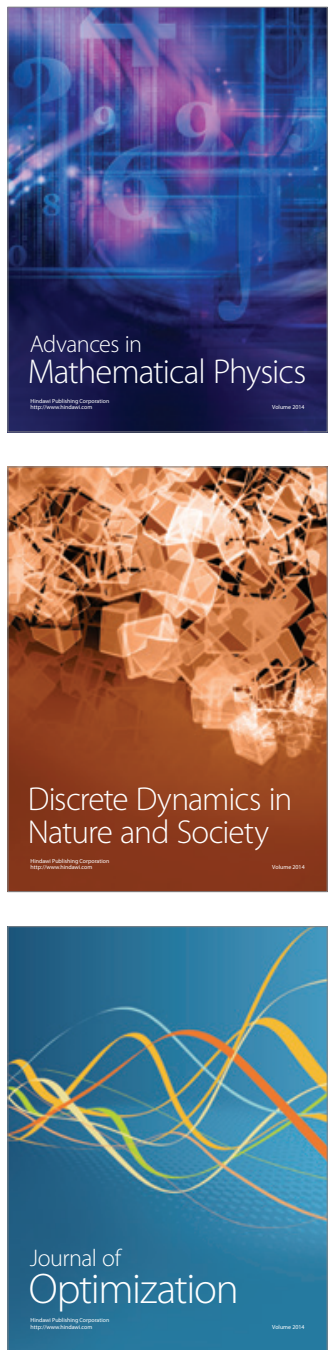